\title{
Towards an optimized flow-sheet for a SANEX demonstration process using centrifugal contactors
}

\author{
By D. Magnusson ${ }^{1,4}$, B. Christiansen ${ }^{1}$, J.-P. Glatz ${ }^{1}$, R. Malmbeck ${ }^{1, *}$, G. Modolo ${ }^{2}$, D. Serrano-Purroy ${ }^{1}$ and C. Sorel ${ }^{3}$ \\ ${ }^{1}$ European Commission, Joint Research Centre, Institute for Transuranium Elements, Postfach 2340, 76125 Karlsruhe, Germany \\ 2 Forschungszentrum Jülich, Institute for Energy Research, Safety Research and Reactor Technology, 52425 Jülich, Germany \\ 3 Commissariat à 1 'Énergie Atomique Valrho (CEA), DRCP/SCPS, BP17171, 30207 Bagnols-sur-Cèze, France \\ ${ }^{4}$ Chalmers University of Technology, Nuclear Chemistry, Department of Chemical and Biological Engineering, Gothenburg, Sweden
}

(Received June 10, 2008; accepted in revised form October 5, 2008)

\section{Extraction / Kinetics / Partitioning / Centrifugal contactor / Minor actinides $/ \mathrm{CyMe}_{4}-B T B P$}

\begin{abstract}
Summary. The design of an efficient process flow-sheet requires accurate extraction data for the experimental set-up used. Often this data is provided as equilibrium data. Due to the small hold-up volume compared to the flow rate in centrifugal contactors the time for extraction is often too short to reach equilibrium $D$-ratios. In this work single stage kinetics experiments have been carried out to investigate the $D$-ratio dependence of the flow rate and to compare this with equilibrium batch experiments for a SANEX system based on $\mathrm{CyMe}_{4}$-BTBP. The first centrifuge experiment was run with spiked solutions while in the second a genuine actinide/lanthanide fraction from a TODGA process was used. Three different flow rates were tested with each set-up. The results show that even with low flow rates, only around 9\% of the equilibrium $D$-ratio (Am) was reached for the extraction in the spiked test and around $16 \%$ in the hot test (the difference is due to the size of the centrifuges). In the hot test the lanthanide scrubbing was inefficient whereas in the stripping both the actinides and the lanthanides showed good results. Based on these results improvements of the suggested flow-sheet is discussed.
\end{abstract}

\section{Introduction}

Partitioning and transmutation (P\&T) scenarios for spent nuclear fuels aim to reduce waste radiotoxicity and volumes [1,2]. The concept for partitioning and recovery of minor actinides, that has been developed within collaborations supported by the European Commission, combines two consecutive separation processes applied on high active raffinate (HAR) from the PUREX process. A group separation of actinides and lanthanides takes place in the first step which relies on the extraction of trivalent elements leaving the remaining fission products in the raffinate. This process is then followed by a SANEX (selective actinide extraction) process in which actinides are separated and recovered from the lanthanides. The current SANEX reference system is

\footnotetext{
*Author for correspondence

(E-mail: rikard.malmbeck@ec.europa.eu).
}

based on the 6,6'-bis(5,5,8,8-tetramethyl-5,6,7,8-tetrahydrobenzo[1,2,4]triazin-3-yl)-[2,2'] bipyridine ( $\mathrm{CyMe}_{4}$-BTBP) extractant $[3,4]$.

As a part of the process development, small-scale reprocessing tests are carried out in which the separation efficiency of the extraction system is demonstrated using simulated HAR spiked with relevant actinides [5,6] and genuine solutions originating from dissolved spent nuclear fuel $[7,8]$. For this purpose, counter current centrifugal contactor systems have been set-up and installed in hot cell facilities. The actual flow-sheet used for the demonstration test is optimised to maximise actinide recovery and to minimise the concentration of impurities in the product fraction. Important parameters are the total number and distribution of contactors between extraction, scrubbing and stripping sections, concentrations (acidity, complexing agents, etc) and flow-rates. Concentration profiles and recoveries are modelled by computer code calculations [9]. The most essential input is accurate distribution data of extracted elements at the conditions used. In this work distribution data of Am, Cm (actinides) and Eu (lanthanides) for the SANEX extraction system have been investigated in two single stage centrifugal contactors of different size. Influence of flow-rates and contactor volumes are discussed as well as the impact on the flow-sheet development and optimisation. Experimental data are compared to computer simulations performed using the PAREX code [9].

\section{Background}

When using centrifugal contactors for solvent extraction processes it is important to investigate the kinetic behaviour of the system. The reason for this is that often high flowrates are used compared to the volume of the centrifuge's mixing chamber. This results in too short residence time for the reagents to be able to reach extraction/back extraction equilibrium.

Extraction data for modelling is often presented as the equilibrium distribution ratios $(D=$ metal concentration in the organic phase divided by the metal concentration in the aqueous phase). Batch experiments with different mixing 


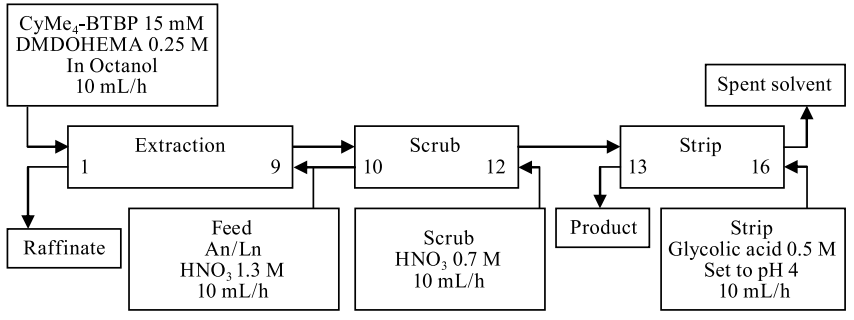

Fig. 1. Proposed flow-sheet for the CyMe4-BTBP demonstration test.

times are used to find the equilibrium time and to express the $D$-ratio as a function of the mixing time.

How far towards equilibrium the extraction reach in each centrifugal contactor depends on the mixing chamber size, flow-rates, mixing efficiency and chemical complex formation rate. To get an idea of how efficient each step is, single centrifuge experiments could be run [10-12] under the same conditions as in the complete system. The apparent $D$-ratios from such an experiment can then be compared with the equilibrium values to determine an efficiency factor.

In this work the efficiency was tested for the 3 steps (extraction, scrub, strip) in the flow-sheet presented in Fig. 1.

The flow-sheet was designed for a demonstration test of a SANEX process, spiked and hot, with the $\mathrm{CyMe}_{4}$-BTBP molecule. The single stage tests were carried out to investigate the efficiency dependence on the flow-rates. Also two different types of centrifugal contactors were tested, one for spiked tests with a smaller mixing chamber and one for hot tests with a larger mixing chamber.

The modelling of the single stage results was based on results from batch experiments, i.e. $D$-values and extraction rate data available for this extraction system.

\section{Experimental}

\subsection{Solutions}

\section{Spiked test}

The organic phase was prepared by first dissolving DMDOHEMA to a concentration of $0.25 \mathrm{M}$ in $n$-octanol. The obtained solution was subsequently equilibrated with $1 \mathrm{M}$ $\mathrm{HNO}_{3}$. The organic mixture was then used to dissolve $\mathrm{CyMe}_{4}$-BTBP (provided by the University of Reading) to a final concentration of $0.015 \mathrm{M}$.

The feed for the extraction test consisted of a solution corresponding to the aqueous phase in the extraction section of a SANEX type process, see Table 1 . The acidity was set to $1.15 \mathrm{M}$ with $\mathrm{HNO}_{3}$.

For the stripping test an aqueous phase of $0.5 \mathrm{M}$ glycolic acid set to $\mathrm{pH} 4$ with ammonia was prepared.

Table 1. Composition of the synthetic SANEX type feed.

\begin{tabular}{lrcccc}
\hline Element & $\mathrm{mg} / \mathrm{L}$ & Element & $\mathrm{mg} / \mathrm{L}$ & Element & $\mathrm{mg} / \mathrm{L}$ \\
\hline $\mathrm{Y}$ & 56 & $\mathrm{Nd}$ & 764 & $\mathrm{Cf}-252$ & tracer \\
$\mathrm{La}$ & 209 & $\mathrm{Sm}$ & 151 & $\mathrm{Am}-241$ & tracer \\
$\mathrm{Ce}$ & 397 & $\mathrm{Eu}$ & 140 & $\mathrm{Cm}-244$ & tracer \\
$\mathrm{Pr}$ & 204 & $\mathrm{Gd}$ & 78 & $\mathrm{Eu}-152$ & tracer \\
\hline
\end{tabular}

Table 2. Concentrations of some nuclides in the undiluted TODGA product.

\begin{tabular}{lccccc}
\hline Nuclide & {$[\mathrm{ppm}]$} & Nuclide & {$[\mathrm{ppm}]$} & Nuclide & {$[\mathrm{ppm}]$} \\
\hline La-139 & 304 & Nd-143 & 124 & Pm-147, & 38 \\
& & & & Sm-147 & \\
Ce-140 & 288 & Nd-144 & 364 & Am-243 & 68 \\
Pr-141 & 257 & Nd-145 & 138 & Cm-244 & 45 \\
Ce-142, & 247 & Nd-146 & 177 & & \\
Nd-142 & & & & & \\
\hline
\end{tabular}

\section{Hot test}

The organic phase was prepared in the same way as in the spiked test.

The feed for the extraction test consisted of the product fraction from a TODGA process [13] diluted two times to compensate for an added scrub flow in a full process. The acidity was set to $1.15 \mathrm{M}$. Table 2 shows the concentrations of some of the most important nuclides in the undiluted TODGA product.

For the scrub test $0.8 \mathrm{M} \mathrm{HNO}_{3}$ was used as aqueous phase and in the strip test the aqueous phase was of the same kind as in the spiked test.

\subsection{Equipment and procedure}

\section{Spiked test}

The experiments were performed in a single stage centrifugal contactor set-up, Fig. 2. The $1 \mathrm{~cm}$ stainless steel centrifugal contactor used was produced by INET, China, while the contactor house was a home made plexiglas copy of the original (which is grouped in four stages and can not be used in single stage experiments). The rotation speed was set to $4200 \mathrm{rpm}$. Two syringe pumps were coupled to feed the organic and aqueous solutions with highly accurate flow-rate.

In the first experiment the extraction performance was tested using three different flow rates as shown in Table 3. The A/O ratio was set to two according to the proposed flow-sheet in Fig. 1. Attainment of the steady state was checked by gamma spectrometric measurements of Am-241 and Eu-152 at the outlets of the contactor. A batch test was also performed to achieve the equilibrium $D$-ratios. A contacting time of $10 \mathrm{~min}$ (vortex mixer) was expected to be sufficient since $5 \mathrm{~min}$ mixing was proven to be enough time to yield equilibrium [3] (the concentrations and mixing are

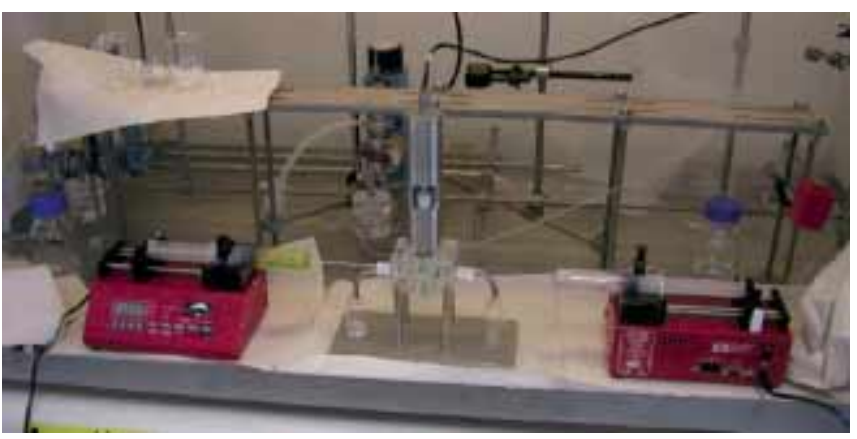

Fig. 2. Single stage centrifugal contactor set-up used in the spiked test. 
Table 3. Flow-rates used in centr. contactor tests.

\begin{tabular}{lcc}
\hline Spiked test & $\begin{array}{c}\text { Organic } \\
\text { phase }[\mathrm{mL} / \mathrm{h}]\end{array}$ & $\begin{array}{c}\text { Aqueous } \\
\text { phase }[\mathrm{mL} / \mathrm{h}]\end{array}$ \\
\hline Extraction 1 & 20 & 40 \\
Extraction 2 & 10 & 20 \\
Extraction 3 & 5 & 10 \\
Strip 1 & 20 & 20 \\
Strip 2 & 10 & 10 \\
Strip 3 & 5 & 5 \\
\hline
\end{tabular}

not completely identical so double contacting time was used in this work).

After the extraction tests all organic solutions were collected. The organic phase was then used to test the stripping kinetics of the centrifuge with the glycolic acid solution as aqueous phase. The flow rate ratio between organic and aqueous phases was set to one and the test was carried out with three different flow rates, see Table 3 . The samples (organic and aqueous) from all the spiked tests were analysed by gamma spectrometry for Am-241 and Eu-152. Additionally, alpha measurements were done for Am-241 and Cm244 in the extraction experiment.

\section{Hot test}

For the hot test the same kind of set-up was used as in the spiked test but here a BXP012 type centrifugal contactor from Rousselet-Robatel was used (inner diameter of rotor $12 \mathrm{~mm}$ ). This centrifuge has a larger mixer volume and therefore offers better kinetic properties. The rotor speed

Table 4. Flow-rates used in centr. contactor tests.

\begin{tabular}{lcc}
\hline Hot test & $\begin{array}{c}\text { Organic } \\
\text { phase }[\mathrm{mL} / \mathrm{h}]\end{array}$ & $\begin{array}{c}\text { Aqueous } \\
\text { phase }[\mathrm{mL} / \mathrm{h}]\end{array}$ \\
\hline Extraction 1 & 17 & 29 \\
Extraction 2 & 10 & 20 \\
Extraction 3 & 6 & 9.6 \\
Scrub 1 & 15 & 15 \\
Scrub 2 & 10 & 10 \\
Scrub 3 & 6 & 6.1 \\
Strip 1 & 15 & 15 \\
Strip 2 & 10 & 10 \\
Strip 3 & 6 & 6.1 \\
\hline
\end{tabular}

was set to around $4000 \mathrm{rpm}$. In the hot cells rotary piston pumps are used.

The flow-rates for the different hot tests are shown in Table 4. The flow-rates were determined by weighting the out-going solutions. A scrubbing step was introduced between the extraction and stripping experiments to acquire information of the scrub kinetics. Samples were taken after a steady state situation was reached.

The samples (organic and aqueous) from all the hot tests were analysed by gamma spectrometry for Am-241 and Eu152. Additionally alpha analysis of Cm-244 was carried out for the extraction samples.

\section{Result and discussion}

\section{Spiked test}

The apparent $D$-values of americium and europium measured during the single centrifuge experiments were compared to the values estimated by the model implemented in the PAREX code (CEA). The results of the kinetic studies and the comparison with calculated $D$-ratios are shown in Table 5. For the extraction it is shown that with decreasing flow-rates, the distribution ratios of the actinides increases slowly. This is expected since the average residence time in the centrifuge increases. However, even at very low flowrates the $D$-ratios were far away from the equilibrium value. This means that the stage efficiency is very low and that the efficient $D$-ratio for Am reaches around 9\% of the equilibrium value at the lowest flow-rate tested (extraction 3). In the stripping test the $D$-ratio decreases with reduced flowrates. This is due to the fact that the metal content enters the contactor by the organic phase instead of the aqueous. The stripping kinetics was also very slow and a distribution ratio of Am below 1 is only reached with the $5 \mathrm{~mL} / \mathrm{h}$ flow-rate. The kinetics was perhaps slower since the loaded organic phase was not scrubbed before stripping and therefore had a higher acid content than suitable. The results of the batch test show however that stripping is possible even if the nitric acid is not scrubbed before. The comparison with the calculated values showed that the effect of the residence time on the apparent $D$-values is quite well simulated for both the extraction and the stripping steps. However, the estimations of the distribution coefficients of americium during the stripping experiments deviate from the experimental values. An explanation could be the increased acidity due to the lack of a previous scrubbing step.

Table 5. Results from the spiked tests, experimental and calculated.

\begin{tabular}{llllclc}
\hline & $\begin{array}{c}D_{\mathrm{Eu}, \gamma} \\
\text { Exp }\end{array}$ & $\begin{array}{c}D_{\mathrm{Eu}} \\
\mathrm{Cal}\end{array}$ & $\begin{array}{c}D_{\mathrm{Am}, \gamma} \\
\mathrm{Exp}\end{array}$ & $\begin{array}{c}D_{\mathrm{Am}, \alpha} \\
\mathrm{Exp}\end{array}$ & $\begin{array}{c}D_{\mathrm{Am}} \\
\mathrm{Cal}\end{array}$ & $\begin{array}{c}D_{\mathrm{Cm}, \alpha} \\
\operatorname{Exp}\end{array}$ \\
\hline Extraction 1 & 0.05 & 0.05 & 0.62 & 0.58 & 0.78 & 0.35 \\
Extraction 2 & 0.08 & 0.08 & 1.5 & 1.3 & 1.5 & 0.73 \\
Extraction 3 & 0.11 & 0.11 & 2.5 & 2.0 & 2.8 & 1.2 \\
Batch extr. & 0.21 & 0.17 & 27 & 25 & 19 & 13 \\
Strip 1 & 1.7 & 2.0 & 5.6 & - & 0.57 & - \\
Strip 2 & 0.65 & 1.0 & 1.8 & - & 0.29 & - \\
Strip 3 & 0.31 & 0.50 & 0.46 & - & 0.15 & - \\
Batch strip & 0.02 & 0.0002 & 0.002 & - & 0.002 & - \\
\hline
\end{tabular}




\section{Hot test}

Table 6 shows the experimental and calculated results from the hot single stage centrifuge tests (sample Scrub 1 was lost). In the extraction test the $D$-ratio increases with lower flow rates as in the spiked test. Due to the size difference somewhat better values were obtained (higher $D$-ratios for the $\mathrm{An}$ ) but none were better than around $16 \%$ of the equilibrium value for Am. $\mathrm{Cm}$ shows about the same values as Am in contrast to the spiked tests. In the scrubbing and stripping tests the $D$-value drops with decreasing flow-rate. Also in these cases the larger centrifuge shows slightly better kinetic properties than the one used in the spiked test. The scrub values for Eu indicate that the back extraction for lanthanides is not efficient in $\sim 0.8 \mathrm{M} \mathrm{HNO}_{3}$, therefore in case of a complete process, low flow-rates and lower acidity in the aqueous phase would be appropriate. Stripping is efficient for both Am and Eu which is different compared to the results obtained in the spiked test. The better kinetics of the larger centrifuge could be responsible for the difference but also the decreased acidity in the organic phase by the scrubbing step plays a role. The comparison of experimental and calculated $D$-ratios for americium and europium, presented in Table 6, shows that except for the stripping experiments, the effect of flow-rate variations on the apparent $D$-ratio is well simulated. Contrary to the experimental behaviour reported in Table 5, the $D$-values measured during the stripping tests for americium and europium are very close. The estimated $D$-ratio of americium and europium are always higher than the experimental ones. Consequently, the calculations for the stripping step of a complete flow-sheet are conservative.

Table 7 shows the stage efficiencies $(E)$ for all the experiments. $E$ is calculated according to Eq. (1)

$$
E=\frac{C_{\text {feed }}-C_{\text {out }}}{C_{\text {feed }}-C_{\text {eq }}},
$$

where $C_{\text {feed }}=$ the metal concentration in the aqueous feed, $C_{\text {out }}=$ the metal concentration in the aqueous product, $C_{\text {eq }}=$ the metal concentration in the aqueous phase at equilibrium in the extraction experiments and $C_{\text {feed }}=$ the metal concentration in the organic feed, $C_{\text {out }}=$ the metal concentration in the organic product, $C_{\mathrm{eq}}=$ the metal concentration

Table 6. Results from the hot tests, experimental and calculated.

\begin{tabular}{lccccc}
\hline & $\begin{array}{c}D_{\mathrm{Eu}} \\
\text { Exp }\end{array}$ & $\begin{array}{c}D_{\mathrm{Eu}} \\
\mathrm{Cal}\end{array}$ & $\begin{array}{c}D_{\mathrm{Am}} \\
\text { Exp }\end{array}$ & $\begin{array}{c}D_{\mathrm{Am}} \\
\mathrm{Cal}\end{array}$ & $\begin{array}{c}D_{\mathrm{Cm}} \\
\text { Exp }\end{array}$ \\
\hline Extraction 1 & 0.11 & 0.08 & 2.3 & 1.6 & 2.4 \\
Extraction 2 & 0.11 & 0.11 & 2.5 & 2.8 & 3.1 \\
Extraction 3 & 0.12 & 0.13 & 4.1 & 4 & 4 \\
Batch extr. & 0.16 & 0.17 & 26 & 19 & 19 \\
Scrub 1 & - & - & - & - & - \\
Scrub 2 & 0.57 & 0.77 & 13 & 15 & - \\
Scrub 3 & 0.38 & 0.5 & 13 & 14 & - \\
Batch scrub & 0.14 & 0.1 & 8.7 & 11 & - \\
Strip 1 & 0.08 & 0.73 & 0.09 & 0.21 & - \\
Strip 2 & 0.04 & 0.53 & 0.04 & 0.16 & - \\
Strip 3 & 0.03 & 0.31 & 0.02 & 0.1 & - \\
Batch strip & 0.01 & 0.0002 & 0.01 & 0.02 & - \\
\hline
\end{tabular}

Table 7. Stage efficiencies [\%] for both the hot and spiked tests.

\begin{tabular}{lcccccc}
\hline & $\begin{array}{c}\text { Eu } \\
\text { spiked }\end{array}$ & $\begin{array}{c}\text { Eu } \\
\text { hot }\end{array}$ & $\begin{array}{c}\text { Am } \\
\text { spiked }\end{array}$ & $\begin{array}{c}\text { Am } \\
\text { hot }\end{array}$ & $\begin{array}{c}\text { Cm } \\
\text { spiked }\end{array}$ & $\begin{array}{c}\text { Cm } \\
\text { hot }\end{array}$ \\
\hline Extr. 1 & 14 & 44 & 25 & 61 & 16 & 63 \\
Extr. 2 & 22 & 37 & 44 & 58 & 29 & 64 \\
Extr. 3 & 32 & 50 & 57 & 75 & 40 & 75 \\
Scrub 1 & - & - & - & - & - & - \\
Scrub 2 & - & 73 & - & 69 & - & - \\
Scrub 3 & - & 83 & - & 73 & - & - \\
Strip 1 & 37 & 95 & 15 & 94 & - & - \\
Strip 2 & 62 & 97 & 36 & 98 & - & - \\
Strip 3 & 78 & 97 & 69 & 99 & - & - \\
\hline
\end{tabular}

in the organic phase at equilibrium in the scrub and strip experiments.

As expected the volume difference of the centrifugal contactors has an impact on the stage efficiency. In the spiked test $E$ is only $57 \%$ for Am in the extraction while in the hot test $E$ reaches $75 \%$. This corresponds to $9 \%$ and $16 \%$ of the equilibrium $D$-ratio for Am in the spiked and the hot tests, respectively. In the hot test similar results were obtained for $\mathrm{Cm}$ while the values in the spiked test were lower. It should be pointed out that in the spiked test a plexiglas contactor house was used which can result in a different mixing compared to the original stainless steel house.

Flow-rates lower than $10 \mathrm{~mL} / \mathrm{h}$ are not convenient to use due to poor pump stability. This means that a $D$-ratio of only 1.5 and 2.5 is available for the Am extraction in the spiked and hot tests, respectively. With the flow-sheet suggested a calculation using constant $D$-ratio of 2.5 in the extraction section indicates only $97 \%$ extracted (which is far from the target value $>99.9 \%$ ). In addition the scrubbing of the lanthanides is inefficient. Improvements of the flow-sheet are therefore necessary and since only 16 stages are available in the hot cells the $D$-ratios have to be optimised. Since the flow-rates can not be decreased, changes in the acidity is the best way to accomplish this. A suggestion is to increase the feed acidity to $2 \mathrm{M}$ and decrease the scrub acidity to $0.5 \mathrm{M}$. This would change the acidity in the extraction section from $\sim 1 \mathrm{M}$ to $\sim 1.3 \mathrm{M}$ and substantially increase the $D$-ratios. The reduced acidity in the scrub is also beneficial for the lanthanide scrubbing. However, these improvements are not sufficient for a demonstration test using the smaller centrifugal contactors.

\section{Conclusions}

The one stage centrifugal contactor investigations have shown that extraction kinetic results obtained in batch tests give information only whether an extraction system is fast or slow. The extraction performance in a complete process will also depend on the equipment used. To optimise a flow-sheet, kinetic data for the given equipment has to be collected under realistic conditions. This ensures more accurate data since correct volumes and mixing in the contactors are assured. In this work, this kind of experiment has shown that only $16 \%$ of the equilibrium $D$-ratio for Am (corresponding to $75 \%$ stage efficiency) was reached in the system investigated. Under conditions suitable for a hot demon- 
stration test (flow-rate $\geq 10 \mathrm{~mL} / \mathrm{h}$ ) even lower efficiencies were obtained. In addition the lanthanide scrubbing was also inefficient whereas the stripping showed good results. Despite this, improvements of the flow-sheet are possible to implement in order to run a successful demonstration test. A simple and efficient way is to increase the acidity in the extraction section. This results in higher $D$-ratios and therefore a more efficient extraction. In the same way the scrubbing could be improved but here by decreasing the acidity which gives a more efficient lanthanide backextraction.

Acknowledgment. Financial support from the European Commission is acknowledged (EUROPART, contract FI6W-CT-2003-508854). M. R. S. Foreman, University of Reading is greatly acknowledged for providing the $\mathrm{CyMe}_{4}$-BTBP.

\section{References}

1. Actinide and Fission Product Partitioning and Transmutation. Status and Assessment Report, OECD-NEA (1999).

2. Accelerator Driven Systems (ADS) and Fast Reactors (FR) in Advanced Nuclear Fuel Cycles - A Comparative Study. OECD/NEA (2002).

3. Geist, A., Hill, C., Modolo, G., Foreman, M. R. S. J., Gompper, K., Weigl, M., Hudson, M. J.: 6,6'-Bis(5,5,8,8-tetramethyl-5,6,7,8tetrahydro-benzo[1,2,4]triazin-3-yl)[2,2']bipyridine, an effective extracting agent for the sepration of americium(III) and curium(III) from the lanthanides. Solv. Extr. Ion Exch. 24(4), 463 (2006).

4. Foreman, M. R. S., Hudson, M. J., Drew, M. G. B., Hill, C., Madic, C.: Complexes formed between the quadridentate, heterocyclic molecules 6,6-bis-(5,6-dialkyl-1,2,4-triazin-3-yl)-2,2bipyridine (BTBP) and lanthanides(III): implications for the partitioning of actinides(III) and lanthanides(III). Dalton Transact. 13, 1581 (2006).

5. Modolo, G., Asp, H., Vijgen, H., Malmbeck, R., Magnusson, D., Sorel, C.: Demonstration of a TODGA-based continuous countercurrent extraction process for the partitioning of actinides from simulated PUREX raffinate. Part II: Centrifugal contactor runs. Solv. Extr. Ion Exch. 26(1), 62 (2008).

6. Modolo, G., Vijgen, H., Serrano-Purroy, D., Christansen, B., Malmbeck, R., Sorel, C., Baron, P.: DIAMEX counter-current extraction process for recovery of trivalent actinides from simulated high active concentrate. Sep. Sci. Technol. 42(3), 439 (2007).

7. Malmbeck, R., Courson, O., Pagliosa, G., Römer, K., Sätmark, B., Glatz, J.-P., Baron, P.: Partitioning of minor actinides from HLLW using the DIAMEX process. Part 2 - "Hot" continuous countercurrent experiment. Radiochim. Acta 88(12), 865 (2000).

8. Serrano-Purroy, D., Baron, P., Christiansen, B., Malmbeck, R., Sorel, C., Glatz, J.-P.: Recovery of minor actinides from HLLW using the DIAMEX process. Radiochim. Acta 93(6), 351-355 (2005).

9. Dinh, B., Mauborgne, B., Baron, P.: Dynamic Simulation of Extraction Operations. Applications in Nuclear Fuel Reprocessing. European Symposium on Computer Aided Process Engineering 2 (ESCAPE2), Toulouse, France, October 5-7 (1992).

10. Sano, Y., Shibata, A., Koizumi, T., Koyama, T., Fox, D., Carrott, M., Taylor, R. J.: U(VI) Back-Extraction Trials for Measurement of U(VI) Mass Transfer Efficiency in Single Stage Centrifugal Contactor. GLOBAL 2005, Tsukuba, Japan, October 9-13 (2005).

11. Rivalier, P., Lanoë, J.-Y.: Development of a new miniature shortresidence-time annular centrifugal solvent extraction contactor for tests of process flowsheets in hot cells. ATALANTE 2000, Avignon, France, October 24-26 (2000).

12. Leonard, R. A., Bernstein, G. J., Ziegler, A. A., Pelto, R. H.: Annular centrifugal contactors for solvent extraction. Sep. Sci. Technol. 15(4), 925 (1980).

13. Magnusson, D., Christansen, B., Glatz, J.-P., Malmbeck, R., Modolo, G., Serrano-Purroy, D., Sorel, C.: Partitioning of Minor Actinides from PUREX Raffinate by the TODGA Process. GLOBAL 2007, Boise, Idaho, USA, September 9-13 (2007). 